\title{
Screening of Domain-specific Target Proteins of Polo-like Kinase 1: Construction and Application of Centrosome/Kinetochore-specific Targeting Peptide
}

\author{
Jae-Hoon $\mathrm{Ji}^{1}$ and Young-Joo Jang* \\ Laboratory of Biochemistry, The School of Dentistry, Dankook University, 29 Anseo-Dong, Cheonan-Si, Chungnam 330-714, Korea \\ ${ }^{1}$ Department of Molecular Biology, Dankook University, Seoul 140-714, Korea
}

Received 26 April 2006, Accepted 20 July 2006

\begin{abstract}
Mammalian polo-like kinase 1 (PIk1) acts at various stages in early and late mitosis. Plk1 localizes at the centrosome and maintains this position through mitosis. Thereafter Plk1 moves to the kinetochore and midbody region, important sites during chromosome separation and cytokinesis. The catalytic domain of PIk1 is in the $\mathrm{N}$ terminus region, whereas the non-catalytic region in the $\mathrm{C}$ terminus of Plk1 has a conserved motif, named the Polobox. This motif is critical for Plk localization. EGFP proteins fused with the $\mathrm{N}$-terminus and $\mathrm{C}$-terminus of Plk1 localize in the nucleus and centrosomes, respectively. The core sequences of the polo-box (50 amino acids) also localize in Plk1 target organelles. To screen for domainspecific target proteins of Plk1, we constructed an $\mathrm{N}$ terminal domain and a tandem repeat polo-box motif, and used them as templates in a yeast two-hybrid screen. The HeLa cell cDNA library indicated several proteins including the centrosome/kinetochore components or regulators, to be characterized as positive clones. Through in vitro protein binding analyses, we confirmed an interaction between these proteins and Plk1. The data reported from this study indicate that the $\mathrm{N}$ - and $\mathrm{C}$ termini of Plk1 may function through recruitment and/or activation of domain-specific target proteins in dividing cells. Additionally, tandem repeats of the conserved core motif of the polo-box are sufficient for targeting and may be useful as a centrosome/kinetochore-specific targeting peptide.
\end{abstract}

Keywords: Centrosome, Kinetochore, Polo-box motif, Pololike kinase 1, Yeast two-hybrid screening

\footnotetext{
*To whom correspondence should be addressed.

Tel: 82-41-550-1681; Fax: 82-41-552-7648

E-mail: yjjang@dku.edu
}

\section{Introduction}

The polo-like kinases (Plks) are a conserved subfamily of serine/threonine protein kinases that play numerous roles during M-phase progression (Glover et al., 1996; Donaldson et al., 2001). Polo kinases are required at several key steps in the process, through initiation of $\mathrm{G} 2 / \mathrm{M}$ by phosphorylation of Cdc25C and mitotic cyclins (Abrieu et al., 1998; Qian et al., 1998; Karaiskou et al., 1999; Toyoshima-Morimoto et al., 2001), centrosome maturation (Sunkel and Glover, 1988; Lane and Nigg, 1996), and the establishment of a bipolar spindle (Ohkura et al., 1995; Lane and Nigg, 1996; Qian et al., 1998). Activation of the anaphase-promoting complex (APC) initiates anaphase and an exit from mitosis (Descombes and Nigg, 1998; May et al., 2002). Microinjection of Plk1 antibodies specific for the C-terminal domain leads to mitotic damage with monopolar spindles, immature centrosomes, and failure of g-tubulin recruitment to the centrosome (Lane and Nigg, 1996). The C-terminal domain of Plk1 inhibits Plk1 kinase activity through intramolecular interaction, and the overexpression of this domain causes centrosome abnormalities (Jang et al., 2002a). Recently, phenotype analysis based on an RNA interference approach revealed the essential function of Plk1 for cell proliferation and viability (Spankuch-Schmitt et al., 2002; Sumara et al., 2004; Van Vugt et al., 2004). These data suggested a dual role for Plk1 in the recruitment and activation of target proteins required for centrosome maturation and bipolar spindle formation.

One of the most intriguing features of Plk1 are the dynamic changes of localization and kinase activity during mitosis. Localization of Plk1 to the centrosome persists from early mitosis until late anaphase (Shirayama et al., 1998; Bahler et al., 1998; Logarinho and Sunkel, 1998; Moutinho-Santos et al., 1999; Mulvihill et al., 1999). Expression of a recombinant Green Fluorescent Protein (GFP) has confirmed the kinetochore/ centromere localization of Plk1 and suggested that Plk1 moves to the kinetochore to possibly regulate chromosome 
and chromatin separation during anaphase (Arnaud et al., 1998). Subsequently, Plk1 is located in the central spindle and the midbody region (Shirayama et al., 1998; Bahler et al., 1998; Logarinho and Sunkel, 1998; Moutinho-Santos et al., 1999; Song et al., 2000).

In mammalian cells, the C-terminus of Plk1 alone directs to centrosomes and midbody (Seong et al., 2002; Jang et al., 2002a). Alignment of the C-terminal regions of several polorelated kinases revealed that there is a motif with significant homology (polo-box) (Hudson et al., 2001), and that the introduction of a mutation in the polo-box disrupted the subcellular localization of Plk1 (Lee et al., 1998). Mutations in the polo-box of Cdc5 (the yeast homolog of Plk1), that do not affect the kinase activity, abolish the ability for localization to the mitotic apparatus in budding yeast (Lee et al., 1998; Song et al., 2000). Plo1 (a Plk1 homolog in fission yeast) interacts with multiple proteins, including cell cycle regulators, in a polo-box-dependent manner (Reynolds and Ohkura, 2003). These data, therefore, suggested that the polo-box is an essential motif for biological activity and the targeting of Plk in mitosis.

Despite a growing list of physiological substrates for Plk1, such as Cdc25, Myt1, cyclin B, NudC, cohesion, and TCTP that have been uncovered recently, the mechanism through which Plk1 regulates bipolar spindle formation, centrosome maturation, or cytokinesis remains unclear (ToyoshimaMorimoto et al., 2002; Sumara et al., 2002; Yarm, 2002; Nakajima et al., 2003; Jackman et al., 2003; Zhou et al., 2003). To better understand various Plk1 functions, it is important to identify Plk1 target proteins and regulators. The results of this study demonstrate several Plk1-interacting proteins through use of the N-terminal and polo-box motif of Plk1 as baits in yeast two-hybrid screens. To avoid the mitotic defect and abnormality by overexpression of the C-terminal domain in cells, we constructed a core polo-box motif which is sufficient to localize target organelles, and used this motif as bait in yeast two-hybrid screens without evidence of cellular toxicities.

\section{Materials and Methods}

Cell culture and mammalian cell transfection. HeLa cells were grown in Dulbecco's modified Eagle's medium supplemented with $10 \%$ fetal bovine serum (HyClone) in a humidified incubator at $37^{\circ} \mathrm{C}$. The standard calcium chloride technique of DNA transfection was carried out except for the substituted use of HEPES-buffered saline (HBS) (Chen and Okayama, 1987; Jang et al., 2002a).

Preparation of plasmids. The polo-box motif (residue 400 to 454) and C-terminal domain (residue 305 to 603) were amplified by PCR from pCMV-FLAG-Plk (wild-type) (Jang et al., 2002a). The N-terminal domain of Plk1 was subcloned into various vectors from pCMV-FLAG-Plk (wild-type) by using SalI and BamHI. The DNA fragments were cloned into pLexA (Jang et al., 2004) for a yeast two-hybrid screen, and into pGEX-4T-2 for the expression of proteins in E. coli. For subcellular localization, they were cloned into the $\mathrm{pEGFP}-\mathrm{Cl}^{\mathrm{TM}}$ (Clontech) vector. All constructs were confirmed by DNA sequencing.

Yeast two-hybrid screening. The N-terminus, C-terminus, and polo-box motifs were cloned into the pLexA in frame with the LexA DNA-binding domain. A yeast strain (EGY48) carrying a reporter gene (LEU2) was cotransformed with a human HeLa cDNA library (clarify, you do not mean the whole library) fused to E. coli acid blob domain B42 and the bait plasmids, pLexA-2xpolo (tandem repeat of amino acid 400-405), pLexA-Plk-N (amino acid 1-401), or pLexA-Plk-C (amino acid 305-603). Transformation was carried out by using the lithium acetate method (Gietz et al., 1992). Leucine-positive colonies were identified by a filter-lifting assay for $\beta$-galactosidase activity. Library-derived DNA was prepared from candidate clones and then analyzed by DNA sequencing.

In vitro pull-down experiments and immunoprecipitation. GST and MBP proteins were expressed in BL21 (DE3) cells. After isopropyl- $\beta$-D-thiogalactoside (IPTG) induction for $5 \mathrm{~h}(0.3 \mathrm{mM}$ final concentration), cells were lysed with PBST buffer (PBS containing $0.5 \%$ Triton X-100). MBP fusion proteins were recovered by their binding to amylose resins (New England Biolab). Amylose resins were incubated with cell extracts which contained the GST fusion proteins. After the pull-down of beads, the bound GST proteins were detected by using anti-GST antibodies. Immunoprecipitation was achieved by lysing the cells with $0.5 \% \mathrm{NP} 40$ buffer containing $50 \mathrm{mM}$ Tris- $\mathrm{HCl}, \mathrm{pH} 7.5$, $100 \mathrm{mM} \mathrm{NaCl}, 0.5 \%$ NP-40, $1 \mathrm{mM}$ DTT, $1 \mathrm{mM} \mathrm{Na} \mathrm{VO}_{4}, 1 \mathrm{mM}$ EDTA, $5 \mathrm{mM}$ EGTA, and a protease inhibitor mixture. To serve as substrates for the kinase assay, the immunoprecipitates were washed once with kinase buffer (50 mM Tris- $\mathrm{HCl}, \mathrm{pH} 7.5,10 \mathrm{mM}$ $\mathrm{MgCl}_{2}, 5 \mathrm{mM}$ DTT, $2 \mathrm{mM}$ EGTA, and $\left.0.5 \mathrm{mM} \mathrm{Na} \mathrm{NO}_{4}\right)$ and analyzed (Jang et al., 2002b).

Immunofluorescence and microscopy. HeLa cells transfected with pEGFP constructs were grown on acid-treated coverslips, that were coated with a fibronectin solution (Sigma, F1141), and fixed with 3\% paraformaldehyde/methanol (Jang et al., 2002a). Coverslips were washed three times in PBS, and used for multiple experiments. DNA in the cells was detected using propidium iodide. Following three final washes with PBS, the signals on the coverslips were detected with a Zeiss LSM510 confocal microscope.

\section{Results and Discussion}

N-terminal construct of Plk1: The N-terminal domain of mammalian PIk1 is localized in the cytosol. To investigate Plk1 functions in cells, we focused on the detection of cellular target proteins of Plk1. We initially separated the total Plk1 (amino acid 1-603, Plk in Fig. 1) into two parts: an N-terminal domain (amino acid 1-401, Plk-N in Fig. 1) and a C-terminal domain (amino acid 305-603, Plk-C in Fig. 1). The N-terminal domain of Plk1 has a conserved catalytic domain for protein kinase activity. Although the N-terminal domain dictates enzyme catalysis, it has been reported that the N-terminal 


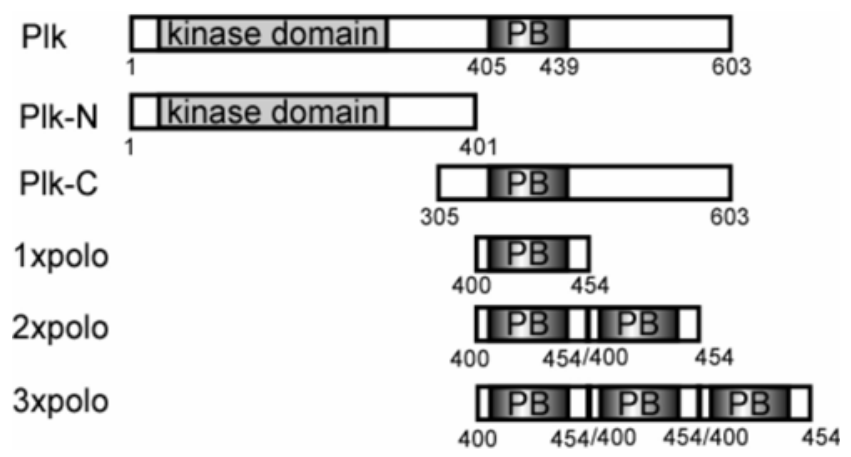

Fig. 1. Construction of the Plk1 domains. The full length of Plk1 (Plk) is 603 amino acids and contains a kinase domain and a polo-box (PB). Actually, two predicted polo-boxes in the Cterminal region of Plk1 (residue 405-439 and 506-598), but we didn't mark the second one in this cartoon. The N-terminal domain (residue 1-401) and the C-terminal domain (residue 305603) were constructed for screening Plk1-interacting proteins (Plk-N \& Plk-C). The 1xpolo indicates a construct containing the core segment of the polo-box (residue 400-454). The tandem repeated constructs of 1 xpolo are indicated as 2 xpolo and 3xpolo.

domain of Plk1 possibly interacts with several other proteins, such as the tubulins (Feng et al., 1999) and the molecular chaperones (Simizu and Osada, 2000), suggesting that the Plk kinase domain is capable of forming stable associations with target proteins. Therefore, we constructed an expression plasmid for the N-terminal domain of Plk1 (amino acid 1401) to screen potential Plk1-interacting proteins (Fig. 1, Plk$\mathrm{N})$. To detect the localization of the N-terminal domain, we fused the domain with Green Fluorescence Protein (GFP) and allowed expression in HeLa cells. Interestingly, this domain protein expression was totally eliminated from the nucleus and was dispersed into the cytosol during interphase (Fig. 2A, upper panels). Even in the mitotic cells, the N-terminal domain of Plk1 was seen to be excluded from the chromosomal DNA region (Fig. 2A, lower panels).

\section{C-terminal construct of Plk1: The core polo-box motif of} mammalian Plk1 mediates its subcellular localization. Multiple roles for Plk1 during mitosis and cytokinesis are supported by the dynamic pattern of its localization.The Plk1 is mainly localized at the centrosomes in interphasic cells. As mitosis progresses, a fraction of Plk1 is redistributed to the microspindle and kinetochore, while the remaining fraction is at the spindle poles. During later stages, Plk1 concentrates in the midbody region (Jang et al., 2002a). Because the subcellular localization of Plk1 is mediated by the C-terminal domain, it is possible that Plk1 interacts with proteins in the centrosomes, kinetochore, and midbody through its Cterminal domain. In our initial Plk1-target screening, we used
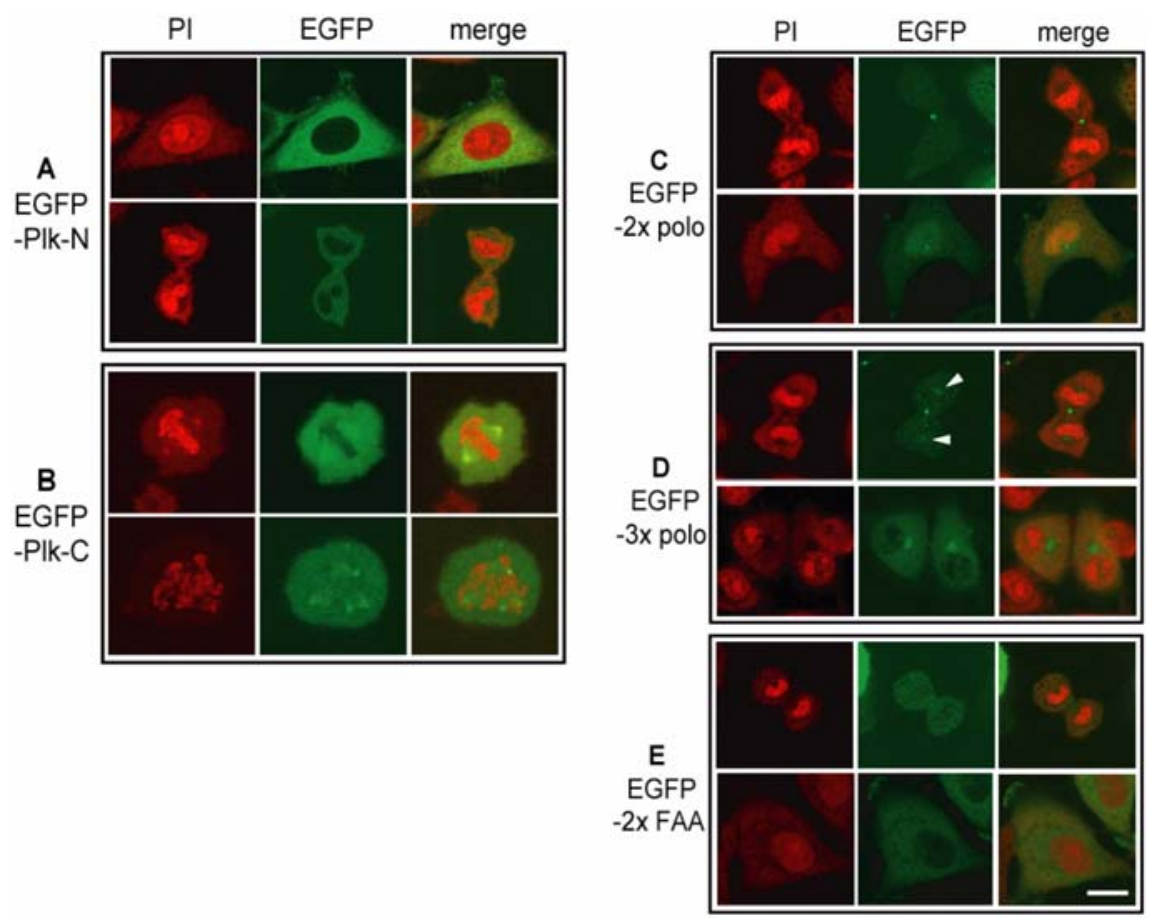

Fig. 2. Subcellular localization of the domain constructs for Plk1 and the polo-box motifs. HeLa cells were transfected with pEGFPPlk-N (A), pEGFP-Plk-C (B), pEGFP-2xpolo (C), and pEGFP-3xpolo (D). As a negative control, three point mutations (W414F, V415A, and L427A) were introduced in 2xpolo, and the mutant construct (pEGFP-2xFAA) was then transfected into HeLa cells (E). Transfectants were permiabilized, fixed, and stained with propidium iodide (PI) for DNA as described in the Materials and Methods. Note that EGFP-FAA does not indicate a specific signal in the centrosome, midbody region, or kinetochores. Arrowheads point to the position of the kinetochores. The scale bar represents $10 \mathrm{~mm}$. 
299 amino acids of the C-terminal domain (amino acid 305603) construct for bait (Fig. 1, Plk-C). Although this construct could be clearly localized in the centrosomes, most of the cells that overexpressed this domain had abnormal centrosomes in their positions and numbers (Fig. 2B, lower panels). A cellular defect based on C-terminus expression is likely caused by inhibition of endogenous Plk1 through a direct interaction of the Plk1 and the C-terminal domain (Jang et al., 2002a). Because of this mitotic abnormality, the C-terminal construct of 299 amino acids needed to be modified. Although there have been many efforts to identify Plk1-target proteins by using the $\mathrm{C}$-terminal domain, few proteins have been reported by the two-hybrid technique, which suggested there could be difficulties in using the C-terminal or full length Plk1. There is a conserved motif in the C-terminal region of Plk1, however, named 'Polo-box', which is important for Plk1 localization. Therefore, we shortened the length of bait in the C-terminal domain into 54 amino acids, which contained the core sequence of the polo-box. When a 54 residue-length (amino acid 400-454) of this motif (Fig. 1, 1xpolo) was fused with GFP and applied for localization, we were able to detect their weak localization in the centrosomes (data not shown). To increase the likelihood of localization, we constructed tandem repeats of this fragment (Fig. 1, 2xpolo \& 3xpolo). Interestingly, tandem repeats clearly localized into centrosomes in both mitotic and interphasic cells (Fig. 2C \& D). In addition to the centrosomes, they also concentrated in the midbody region. Moreover, by using the tandem repeats of the polo core motif, we detected the kinetochore region, which is a structure for chromosome segregation that also contains the anaphase promoting complex (Fig. 2D). These data suggested that the core of the polo-box motif is sufficient to localize to Plk1 target organelles. When several point mutations were introduced into this motif (e.g. W414F/V415A/L427A), the subcellular localization of the polo-box motif was undetectable (Fig. 2E). These findings coincide with previous reports that a Plk1 containing a frame shift mutation in the polo-box did not localize properly (Lee et al., 1998). Thus, these tandem repeat polo-box constructs were used for target protein screening and in vitro binding experiments.

Yeast two-hybrid screening for Plk1-target proteins by using the N-terminus and polo-box motif. The N-terminal domain of Plk1 (Plk-N) and a tandem repeat of the polo-box motif (2xpolo) were cloned into the pLexA vector following the LexA protein coding region. The cDNAs from HeLa cells were ligated into the pJG4-5 vector and fused with B42 protein into the N-terminus. Two plasmid constructs were introduced into yeast strain EGY48, which has two reporters: LEU2 and LacZ. These reporter genes were regulated by the LexA-B42 protein complex. In the first round of screening, the colonies grown as $\mathrm{Leu}^{+}, \mathrm{His}^{+}$, and $\mathrm{Trp}^{+}$were selected: 220 positive colonies from the screening by 2 xpolo and 125 positive colonies from the screening by Plk-N. For the second-round screening, the positive colonies from the $1^{\text {st }}$ screening were applied to a $\beta$-galactosidase filter assay. Finally, 71 of the 220 colonies and 48 of the 125 colonies from the screening by $2 x p o l o$ and Plk-N were shown as strong blue signals in the filter assay. The plasmids containing cDNA fragments were isolated from these positive colonies and characterized by sequencing. As expected, there were many false positives. In the results of the 2xpolo-screening, (71 cases) 12 were ribosomal proteins (16.9\%), and 16 were metabolic enzymes such as enolase and dehydrogenase (22.5\%). Similarly, in the Plk-N-screening (48 cases), 11 were ribosomal proteins $(22.9 \%)$, and 9 were metabolic enzymes (18.8\%). The elongation factor-1 (EF-1) was a protein frequently captured as a false-positive, and this protein was also screened as $5.6 \%$ ( 4 of 71 ) and $4.2 \%$ ( 2 of 48 ) of the total cases from the $2 x p o l o$ and Plk-N screenings, respectively. Interestingly, $\alpha-(1$ of 71$)$ and $\beta$-tubulins ( 3 of 71$)$ interacted with the polo-box motif of Plk1 (data not shown). Previously, Feng et al. (1999) reported that the tubulins associated with both the $\mathrm{N}$ - and C-terminal domains of Plk1 in vitro. Our data coincided with the notion that the polo-box is the essential motif for the interaction of tubulins with the C-terminal domain of Plk1, however, we were unable to verify tubulin clones in the Plk-N screening.

The representative positive clones in the Plk-N and 2xpolo screening are shown in Tables 1-1 and 1-2. The clones that contained the DNA fragment of the same genes are selected in this screening. To investigate whether their interactions were dependent on vectors, cDNA fragments of the positive clones were transferred to the pLexA vector from pJG4-5, and both the Plk-N and 2xpolo were cloned into the pYesTrp vector that contained the B42 domain. The interactions between clones were analyzed by both liquid and filter assays for $\beta$ galactosidase activity. Although the vectors differed, cDNA from positive clones interacted with Plk-N- or 2xpolo constructs, and their associations were over 40 times stronger than the negative control, suggesting that these proteins interact with Plk-N or 2xpolo independent of the vectors (Table 2).

To investigate whether these interactions were domainspecific, two baits were exchanged with each other, and their cross binding activity analyzed. While the PPIA and CCT clones were screened out as Plk-N interacting clones, they nonetheless interacted with the polo-box motif (Table 3). Conversely, there was no cross activity in the polo-box interacting clones.

An in vitro protein interaction experiment was conducted that also supported their interaction. Two baits and cDNA fragments of positive clones were transferred to $E$. coli expression vectors, pGEX and pMAL, respectively. After induction by treatment with IPTG, the MBP-fusion proteins were purified from cell extracts by using MBP-beads. After induction of the GST-fusion baits, cell extracts were prepared and incubated with the purified MBP-fusion proteins. GST antibodies were used to detect baits associated with MBPproteins. Four representative MBP-proteins were interacted with both 2 xpolo and 3xpolo as shown in Fig. 3A. The 


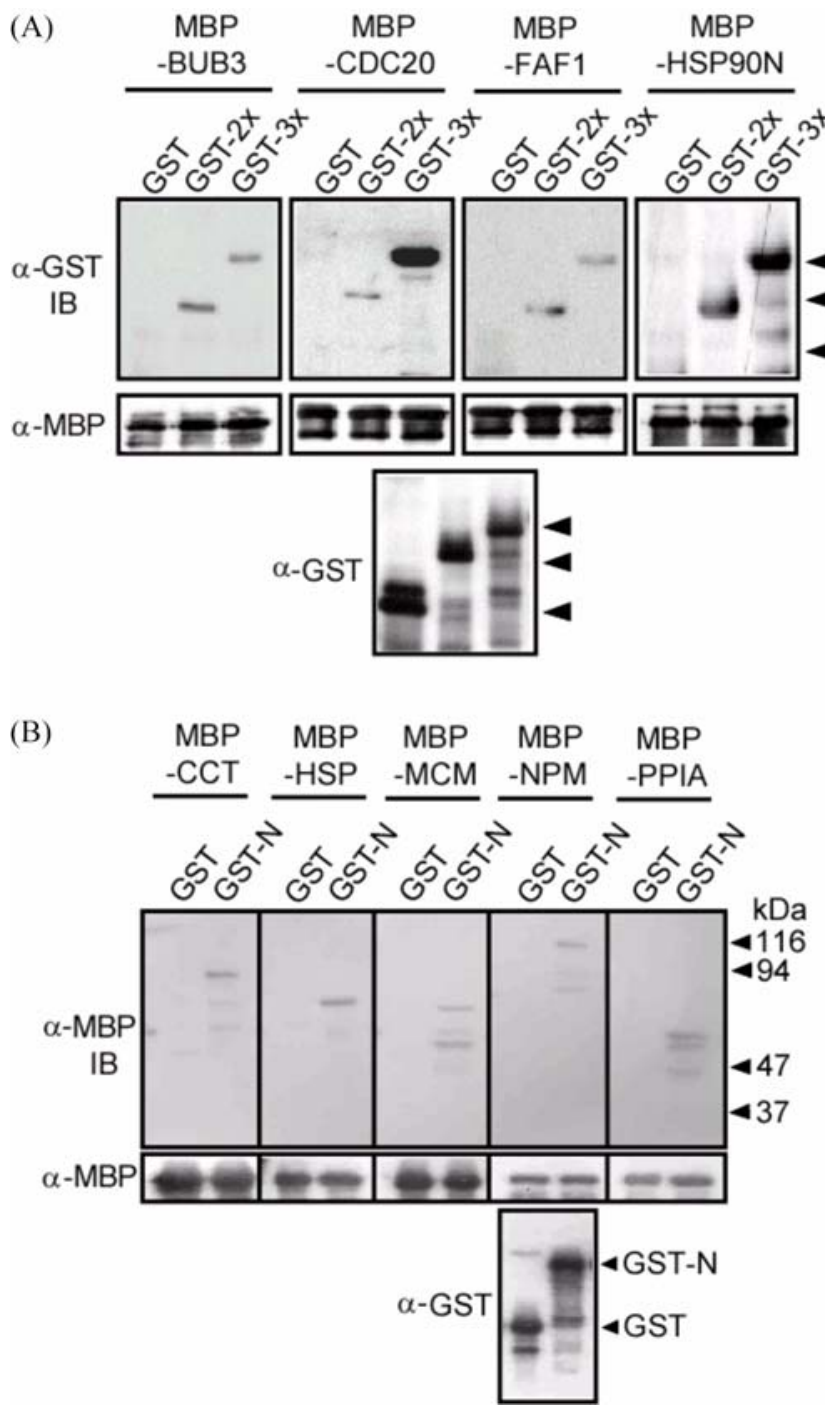

Fig. 3. N-terminal domain and polo-box interactions with proteins as characterized in vitro. The proteins from the positive clones were fused with MBP, and both polo-boxes (A) and the N-terminus of Plk1 (B) were fused with GST. (A) Cell extracts of GST-polo-boxes were incubated with the MBP-purified proteins and the pull-downs were probed by Western blotting with anti-GST antibodies (upper panel, $\alpha$-GST IB). The used amounts of MBP-fusion protein purified and GST-baits in cell extracts were estimated by anti-MBP and anti-GST antibodies, respectively (middle and lower panels). (B) Cell extracts of MBP-fusion clones were incubated with the GST-purified Plk-N and the pull-downs were probed by Western blotting with antiMBP antibodies (upper panel, $\alpha$-MBP IB). The used amounts of MBP-proteins in cell extracts and GST-Plk-N purified were estimated by anti-MBP and anti-GST antibodies, respectively (middle and lower panels).

interactions between both MBP-Cdc20 or -Hsp90N and GST$3 x p o l o$ were stronger than identified for GST-2xpolo (Fig. $3 \mathrm{~A}$ ), which suggested that the three copy tandem repeats of the polo-box has a stronger affinity on the target proteins than
Table 1-1. Representative positive clones interacted with the Nterminus of Plk1. After DNA sequencing, the length of insert cDNA fragments was indicated as 'clone length'. Some clones were characterized as full length ORF (PPIA, protein kinase C inhibitor-1, and nucleophosmin).

\begin{tabular}{|l|r|r|}
\hline $\begin{array}{c}\text { Table 1-1 } \\
\text { N-term-interacting clones }\end{array}$ & $\begin{array}{c}\text { full length } \\
\text { (total amico acids) }\end{array}$ & $\begin{array}{c}\text { clone length }{ }^{\star} \\
\text { (start - end) }\end{array}$ \\
\hline $\begin{array}{r}\text { Peptidylpropyl isomerase A } \\
\text { (PPIA) }\end{array}$ & 165 amino acids & $1-165$ \\
$\begin{array}{c}\text { Minichromosome maintenance } \\
\text { protein 3CM3) }\end{array}$ & 808 amino acids & $682-808$ \\
Protein kinase C inhibitor 1 & 126 amino acids & $1-126$ \\
Chaperonin CCT & 539 amino acids & $105-539$ \\
putative cell cycle control protein & 527 amino acids & $1-500$ \\
Heat shock protein 90 & 724 amino acids & $501-724$ \\
Nucleophosmin & 294 amino acids & $1-294$ \\
\hline
\end{tabular}

Table 1-2. Representative positive clones interacted with $2 x p o l o$. After DNA sequencing, the length of insert cDNA fragments was indicated as 'clone length'.

\begin{tabular}{|l|c|c|}
\hline $\begin{array}{c}\text { Table 1-2 } \\
\text { Polo Box-interacting clones }\end{array}$ & $\begin{array}{c}\text { full length } \\
\text { (total amico acids) }\end{array}$ & $\begin{array}{c}\text { clone length* } \\
\text { (start - end) }\end{array}$ \\
\hline Cell cycle protein CDC20 & 609 amino acids & $110-609$ \\
RanGTPase & 243 amino acids & $46-243$ \\
$\begin{array}{l}\text { Budding uninhibited } \\
\text { by benzimidazoles 3 (BUB3) }\end{array}$ & 328 amino acids & $1-162$ \\
Fas-Associated factor 1 (FAF1) & 650 amino acids & $448-650$ \\
BCL2 homolog MCL1 & 350 amino acids & $186-350$ \\
$\alpha$-catenin & 906 amino acids & $737-906$ \\
Heat shock protein 90 & 724 amino acids & $1-258$ \\
HsCdc18 & 560 amino acids & $202-560$ \\
\hline
\end{tabular}

the two copy repeat constructs. Interactions between five representative Plk-N-interacting proteins and GST-Plk-N were shown in vitro (Fig. 3B). After induction of five MBP-fusion proteins, cell extracts were prepared and incubated with the purified GST-fusion baits. MBP antibodies were used to detect baits associated with GST-Plk-N. CCT (chaperones) containing TCP-1, 110 residue to the end), C-terminal of HSP90 (500 residue to the end), MCM3 (DNA replication licensing factor, 633 residue to the end), and full lengths of nucleophosmin and PPIA (peptidyl-prolyl cis-trans isomerase A), which consisted of the same constructs with two-hybrid clones, were provided for interaction with GST-Plk-N in vitro. Other MBP-fusion proteins were also interacted with GST fusion bait (data not shown).

Interestingly, both the $\mathrm{N}$-terminal and the $\mathrm{C}$-terminal domains of Hsp90 interacted with Plk1. The C-terminal domain of Hsp90 associated with the N-terminal domain of Plk1, 


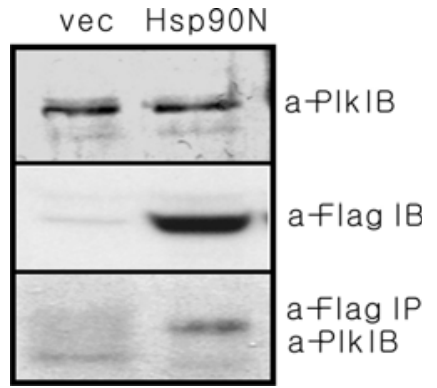

Fig. 4. Endogenous Plk1 interacts with $\mathrm{Hsp} 90 \mathrm{~N}$ in mitotic HeLa cells in vivo. HeLa cells were transfected with FLAG-tagged Hsp90N (amino acid 1-260) (Hsp90N) and vector only (vec). After $24 \mathrm{~h}$ in transfection, nocodazole was added for mitotic arrest for a $16 \mathrm{~h}$ period and the transfectants collected. FLAGtagged proteins were immunoprecipitated by anti-FLAG antibodies. To detect endogenous Plk1 interacted with Hsp90N proteins, immunoprecipitates were probed by Western blotting with antiPlk1 antibodies (lower panel, $\alpha$-FLAG IP/ $\alpha-P l k$ IB). The endogenous Plk1 and FLAG-Hsp90N in mitotic HeLa cell extracts were estimated by anti-Plk1 antibody and anti-FLAG antibody, respectively (upper and middle panels).

whereas the N-terminal half of Hsp90 associated with the polo-box motif (Table 1). Previously, Simizu et al. (2000) had reported that Hsp90 is a target protein of Plk1 in mammalian cells and had characterized the Hsp90-interacting domains of Plk1. They suggested that molecular chaperones could be targeted to Plk1 and could regulate Plk1 stability. As expected, endogenous Plk1 interacted with the N-terminus of Hsp90 (amino acid 1-260) in mitotic HeLa cells (Fig. 4). In this regard, Liu et al. (2005) reported recently that the molecular chaperone protein (CCT) is necessary for Plk1 maturation. In our results, CCT as well as Hsp90, was characterized as one of the target proteins of Plk1, thereby indicating that the tandem repeat construct of the polo box is an excellent bait for interacting with cellular target proteins.

Plk1 localizes in various cell organelles during mitosis such as centrosome, kinetochore, and the midbody region. As shown in Fig. 2, the tandem repeats of the polo-box (2xpolo and 3 xpolo) concentrated in these organelles. According to subcellular localization, we had predicted that the tandem repeat motif would be useful for the screening of target proteins, components of these organelles. Indeed, RanGTPase, $\mathrm{Cdc} 20$ and Bub3 were characterized as polo-box-interacting proteins (Table 1-2). These data strongly suggest direct evidence for Plk1 to localize in centrosomes and the kinetochore and thereby perhaps regulate these components. Recently, we reported that an association between Plk1 and Ran protein occurred in the mitotic centrosome region (Jang et al., 2004). In addition to the centrosome, Plk1 might localize in the kinetochore region through interactions with $\mathrm{Cdc} 20$ and Bub3. The regulation of these proteins by Plk1 in centrosome maturation or chromosome segregation is planned for further study.
Table 2. Confirmation of positive clones by the exchange of vectors. The DNA fragments of bait were cloned into the pJG45 vector, which were used for cDNA expression, and the inserts of positive clones were transferred into the pLexA vector. The indicated plasmids were transfected into the yeast strain (EGY48) and individual cotransformants were patched onto $\mathrm{SD} /$ $\mathrm{gal} / \mathrm{raf} /$-His/-Trp/-Leu/-Ura to select for all plasmids expressing interactive hybrid proteins (see Materials and Methods), $\beta$-gal lift assays were performed ( $\beta$-gal filter assay). The blue color indicates a positive signal in the assay. For quantitative analysis of protein interactions, colonies were subjected to a liquid $\beta$ galactosidase assay as described previously (relative $\beta$-gal activity). The N-terminal domain- and 2xpolo-interacting clones are indicated in (A) and (B), respectively. The intensity of blue color was indicated as '+'; a negative signal on the $\beta$-gal lift assay was indicated as ' - '.

\begin{tabular}{|c|c|c|c|}
\hline A Bait : $\begin{array}{c}\text { N-terminus } \\
(1-401)\end{array}$ & $\begin{array}{c}\text { relative } \\
\beta \text {-gal activity }\end{array}$ & $\begin{array}{l}\beta \text {-gal } \\
\text { filter } \\
\text { assay }\end{array}$ & \\
\hline pYes + pLex & 1.00 & & - \\
\hline pYes-PIk-N + pLex & 1.12 & & - \\
\hline pYes-PIk-N + pLex-PPIA & 40.23 & & + \\
\hline pYes-Plk-N + pLex-MCM3 & 39.00 & & + \\
\hline pYes-PIk-N + pLex-PKCl1 & 42.66 & & + \\
\hline pYes-Plk-N + pLex-CCT & 39.35 & & + \\
\hline pYes-PIk-N + pLex-CCCP & 32.00 & & + \\
\hline pYes-PIk-N + pLex-HSP90C & 39.00 & & + \\
\hline pYes-Plk-N + pLex-NPM & 42.05 & & + \\
\hline $\begin{array}{l}\text { Bait : } 2 x \text { Polo-Box } \\
(400-454 / 400-454)\end{array}$ & $\begin{array}{c}\text { relative } \\
\beta \text {-gal activity }\end{array}$ & $\begin{array}{l}\beta \text {-gal } \\
\text { filter } \\
\text { assay } \\
\end{array}$ & \\
\hline pYes + pLex & 1.00 & & - \\
\hline pYes-Polo + pLex & 0.95 & & - \\
\hline pYes-Polo + pLex-CDC20 & 42.66 & & + \\
\hline pYes-Polo + pLex-Ran & 35.00 & & + \\
\hline pYes-Polo + pLex-BUB3 & 57.23 & & + \\
\hline pYes-Polo + pLex-FAF1 & 32.35 & 8 & + \\
\hline pYes-Polo + pLex-MCL1 & 32.00 & $\infty$ & + \\
\hline pYes-Polo + pLex- $\alpha$-catenin & 39.00 & 2 & + \\
\hline pYes-Polo + pLex-HSP9ON & 41.05 & \&. & + \\
\hline pYes-Polo + pLex-hCDC18 & 34.05 & -8 & + \\
\hline
\end{tabular}

A group of the identified target proteins were apoptosisrelated, which interacted with the polo-box motif (Table 1-2, FAF1 and MCL1). To date, there have been several reports regarding Plk1 function in apoptosis. Thus, when Plk1 was silenced in cancer cells by small interference RNA (SiRNA), the cells ceased to proliferate and apoptosis was induced (Liu and Erikson, 2003). Because Plk1 is a key factor for progression of mitosis, it was predicted that cell proliferation would be inhibited by Plk1 silencing. We have in addition provided evidence that Plk1 may regulate the apoptosis pathway by a direct interaction with several apoptotic factors. 
Table 3. Confirmation of domain specificity of the positive clones. The pJG4-5 constructs of the N-terminal domain-interacting proteins were applied to two-hybrid by using pLexA-2xpolo as bait. Plasmid constructs of polo-box-interacting proteins were applied to two-hybrid by using pLexA-Plk-N. Individual cotransformants were patched and $\beta$-gal lift assays were performed. '+' indicates a positive blue signal. '-' indicates a negative signal. PPIA and CCT were interacted with both the Plk-N and 2xpolo components.

\begin{tabular}{|l|c|c|}
\hline \multirow{2}{*}{ Clones } & \multicolumn{2}{c|}{ Bait } \\
\cline { 2 - 3 } \multicolumn{1}{|c}{ protein 3 (MCM3) } & + & - \\
\hline Peptidylpropyl isomerase A (PPIA) & + & 2xpolo \\
Minichromosome maintenance & + \\
Protein kinase C inhibitor 1 & + & - \\
Chaperonin CCT & + & + \\
Putative cell cycle control protein & + & - \\
Heat shock protein 90 C & + & - \\
Nucleophosmin & + & - \\
Cell cycle protein CDC20 & - & + \\
RanGTPase & - & + \\
Budding uninhibited \\
by benzimidazoles 3 (BUB3) & - & + \\
Fas-Associated factor 1 (FAF1) & - & + \\
BCL2 homolog MCL1 & - & + \\
$\alpha$-catenin & - & + \\
Heat shock protein 90 N & - & + \\
HsCdc18 & - & + \\
\hline
\end{tabular}

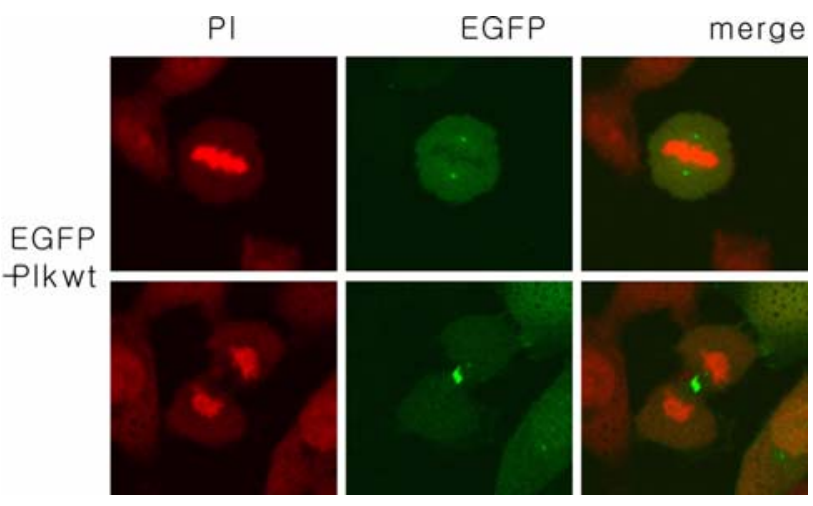

Supplementary data-1. Subcellular localization of full length of Plk1.

In conclusion, we have identified two subjects which are important for the study of intracellular functions of Plk1. First, we have constructed a centrosome/kinetochore-specific targeting peptide with applications to the screening of cellular components regulated by Plk1. Importantly, this peptide is no effect on cell growth, whereas the C-terminal domain of Plk1 induces mitotic chaos. Therefore, this specific motif may be useful as a cellular marker of the centrosome/kinetochore as well as the midbody region and without toxic effects. Secondly, we identified several possible target proteins of Plk1 by yeast-two hybrid screening. Various targets screened by using the Nterminal and polo-box motif as baits provide potential pathways for the further study of Plk1 functions within cells.

Acknowledgment We thank Prof. Chae, S.K. for the pLexA plasmid for two-hybrid. This work was supported by grant R01-2004-000-10399-0 (2006) from the Korea Science and Engineering Foundation.

\section{References}

Abrieu, A., Brassac, T., Galas, S., Fisher, D., Labbe, J. C. and Doree, M. (1998) The Polo-like kinase Plx1 is a component of the MPF amplification loop at the G2/M-phase transition of the cell cycle in Xenopus eggs. J. Cell Sci. 111, 1751-1757.

Arnaud, L., Pines, J. and Nigg, E. A. (1998) GFP tagging reveals human Polo-like kinase 1 at the kinetochore/centromere region of mitotic chromosomes. Chromosoma 107, 424-429.

Bahler, J., Steever, A. B., Wheatley, S., Wang, Y., Pringle, J. R., Gould, K. L. and McCollum, D. (1998) Role of polo kinase and Mid1p in determining the site of cell division in fission yeast. J. Cell Biol. 143, 1603-1616.

Chen, C. and Okayama, H. (1987) High-efficiency transformation of mammalian cells by plasmid DNA. Mol. Cell Biol. 7, 27452752.

Descombes, P. and Nigg, E. A. (1998) The polo-like kinase Plx1 is required for $\mathrm{M}$ phase exit and destruction of mitotic regulators in Xenopus egg extracts. EMBO J. 17, 1328-1335.

Donaldson, M. M., Tavares, A. A., Hagan, I. M., Nigg, E. A. and Glover, D. M. (2001) The mitotic roles of Polo-like kinase. $J$. Cell Sci. 114, 2357-2358.

Feng, Y., Hodge, D. R., Palmieri, G., Chase, D. L., Longo, D. L. and Ferris, D. K. (1999) Association of polo-like kinase with alpha-, beta- and gamma-tubulins in a stable complex. Biochem. J. 339, 435-442.

Gietz, D., St Jean, A., Woods, R. A. and Schiestl, R. H. (1992) Improved method for high efficiency transformation of intact yeast cells. Nucleic Acids Res. 20, 1425.

Glover, D. M., Ohkura, H. and Tavares, A. (1996) Polo kinase: the choreographer of the mitotic stage? J. Cell Biol. 135, 16811684.

Hudson, J. W., Kozarova, A., Cheung, P., Macmillan, J. C., Swallow, C. J., Cross, J. C. and Dennis, J. W. (2001) Late mitotic failure in mice lacking Sak, a polo-like kinase. Curr. Biol. 11, 441-446.

Jackman, M., Lindon, C., Nigg, E. A. and Pines, J. (2003) Active cyclin B1-Cdk1 first appears on centrosomes in prophase. Nat. Cell Biol. 5, 143-148.

Jang, Y. J., Lin, C. Y., Ma, S. and Erikson, R. L. (2002a) Functional studies on the role of the C-terminal domain of mammalian polo-like kinase. Proc. Natl. Acad. Sci. U.S.A 99, 1984-1989.

Jang, Y. J., Ma, S., Terada, Y. and Erikson, R. L. (2002b) Phosphorylation of threonine 210 and the role of serine 137 in the regulation of mammalian polo-like kinase. J. Biol. Chem. 
277, 44115-44120.

Jang, Y. J., Ji, J. H., Ahn, J. H., Hoe, K. L., Won, M., Im, D. S., Chae, S. K., Song, S. and Yoo, H. S. (2004) Polo-box motif targets a centrosome regulator, RanGTPase. Biochem. Biophys. Res. Commun. 325, 257-264.

Karaiskou, A., Jessus, C., Brassac, T. and Ozon, R. (1999) Phosphatase 2A and polo kinase, two antagonistic regulators of cdc25 activation and MPF auto-amplification. J. Cell Sci. 112, 3747-3756.

Lane, H. A. and Nigg, E. A. (1996) Antibody microinjection reveals an essential role for human polo-like kinase 1 (Plk1) in the functional maturation of mitotic centrosomes. J. Cell Biol. 135, 1701-1713.

Lee, K. S., Grenfell, T. Z., Yarm, F. R. and Erikson, R. L. (1998) Mutation of the polo-box disrupts localization and mitotic functions of the mammalian polo kinase Plk. Proc. Natl. Acad. Sci. USA 95, 9301-9306.

Liu, X. and Erikson, R. L. (2003) Polo-like kinase (Plk)1 depletion induces apoptosis in cancer cells. Proc. Natl. Acad. Sci. USA 100, 5789-5794.

Liu, X., Lin, C. Y., Lei, M., Yan, S., Zhou, T. and Erikson, R. L. (2005) CCT chaperonin complex is required for the biogenesis of functional Plk1. Mol. Cell. Biol. 25, 4993-5010.

Logarinho, E. and Sunkel, C. E. (1998) The Drosophila POLO kinase localises to multiple compartments of the mitotic apparatus and is required for the phosphorylation of MPM2 reactive epitopes. J. Cell Sci. 111, 2897-2909.

May, K. M., Reynolds, N., Cullen, C. F., Yanagida, M. and Ohkura, H. (2002) Polo boxes and Cut23 (Apc8) mediate an interaction between polo kinase and the anaphase-promoting complex for fission yeast mitosis. J. Cell Biol. 156, 23-28.

Moutinho-Santos, T., Sampaio, P., Amorim, I., Costa, M. and Sunkel, C. E. (1999) In vivo localisation of the mitotic POLO kinase shows a highly dynamic association with the mitotic apparatus during early embryogenesis in Drosophila. Biol. Cell 91, 585-596.

Mulvihill, D.P., Petersen, J., Ohkura, H., Glover, D. M. and Hagan, I. M. (1999) Plo1 kinase recruitment to the spindle pole body and its role in cell division in Schizosaccharomyces pombe. Mol. Biol. Cell 10, 2771-2785.

Nakajima, H., Toyoshima-Morimoto, F., Taniguchi, E. and Nishida, E. (2003) Identification of a consensus motif for Plk (Polo-like kinase) phosphorylation reveals Myt1 as a Plk1 substrate. J. Biol. Chem. 278, 25277-25280.

Ohkura, H., Hagan, I. M. and Glover, D. M. (1995) The conserved Schizosaccharomyces pombe kinase plo1, required to form a bipolar spindle, the actin ring, and septum, can drive septum formation in G1 and G2 cells. Genes Dev. 9, 10591073.

Qian, Y. W., Erikson, E., Li, C. and Maller, J. L. (1998) Activated polo-like kinase Plx1 is required at multiple points during mitosis in Xenopus laevis. Mol. Cell. Biol. 18, 4262-4271.
Reynolds, N. and Ohkura, H. (2003) Polo boxes form a single functional domain that mediates interactions with multiple proteins in fission yeast polo kinase. J. Cell Sci. 116, 13771387.

Seong, Y. S., Kamijo, K., Lee, J. S., Fernandez, E., Kuriyama, R., Miki, T. and Lee, K. S. (2002) A spindle checkpoint arrest and a cytokinesis failure by the dominant-negative polo-box domain of Plk1 in U-2 OS cells. J. Biol. Chem. 277, 32282-32293.

Shirayama, M., Zachariae, W., Ciosk, R. and Nasmyth, K. (1998) The Polo-like kinase Cdc5p and the WD-repeat protein Cdc20p/fizzy are regulators and substrates of the anaphase promoting complex in Saccharomyces cerevisiae. EMBO J. 17, 1336-1349.

Simizu, S. and Osada, H. (2000) Mutations in the Plk gene lead to instability of Plk protein in human tumour cell lines. Nat. Cell Biol. 2, 852-854.

Song, S., Grenfell, T. Z., Garfield, S., Erikson, R. L. and Lee, K. S. (2000) Essential function of the polo box of Cdc5 in subcellular localization and induction of cytokinetic structures. Mol. Cell. Biol. 20, 286-298.

Spankuch-Schmitt, B., Bereiter-Hahn, J., Kaufmann, M., and Strebhardt, K. (2002). Effect of RNA silencing of polo-like kinase-1 (PLK1) on apoptosis and spindle formation in human cancer cells. J. Natl. Cancer Inst. 94, 1863-1877.

Sumara, I., Vorlaufer, E., Stukenberg, P. T., Kelm, O., Redemann, N., Nigg, E. A. and Peters, J. M. (2002) The dissociation of cohesin from chromosomes in prophase is regulated by Pololike kinase. Mol. Cell 9, 515-525.

Sumara, I., Gimenez-Abian, J. F., Gerlich, D., Hirota, T., Kraft, C., De La Torre, C., Ellenberg, J., and Peters, J. M. (2004) Roles of Polo-like kinase 1 in the assembly of functional mitotic spindles. Curr. Biol. 14, 1712-1722.

Sunkel, C. E. and Glover, D. M. (1988) polo, a mitotic mutant of Drosophila displaying abnormal spindle poles. J. Cell Sci. 89, 25-38.

Toyoshima-Morimoto, F., Taniguchi, E., Shinya, N., Iwamatsu, A. and Nishida, E. (2001) Polo-like kinase 1 phosphorylates cyclin B1 and targets it to the nucleus during prophase. Nature 410, 215-220.

Toyoshima-Morimoto, F., Taniguchi, E. and Nishida, E. (2002) Plk1 promotes nuclear translocation of human $\mathrm{Cdc} 25 \mathrm{C}$ during prophase. EMBO Rep. 3, 341-348.

Van Vugt, M. A., Van De Weerdt, B. C., Vader, G., Janssen, H., Calafat, J., Klompmaker, R., Wolthuis, R. M. and Medema, R. H. (2004) Polo-like kinase-1 is required for bipolar spindle formation but is dispensable for $\mathrm{APC} / \mathrm{Cdc} 20$ activation and initiation of cytokinesis. J. Biol. Chem. 279, 36841-36854.

Yarm, F. R. (2002) Plk phosphorylation regulates the microtubulestabilizing protein TCTP. Mol. Cell. Biol. 22, 6209-6221.

Zhou, T., Aumais, J. P., Liu, X., Yu-Lee, L. Y. and Erikson, R. L. (2003) A role for Plk1 phosphorylation of NudC in cytokinesis. Dev. Cell 5, 127-138. 